\title{
Fractional dynamics of a system with particles subjected to impacts
}

\author{
J.A. Tenreiro Machado
}

\section{A B S T R A C T}

This paper studies the dynamics of a system composed of a collection of particles that exhibit collisions between them. Several entropy measures and different impact conditions of the particles are tested. The results reveal a Power Law evolution both of the system energy and the entropy measures, typical in systems having fractional dynamics.

Keywords:

Fractional calculus

Power Law

Entropy

Non-linear dynamics

Complex systems

\section{Introduction}

Fractional Calculus (FC) is a generalization of the classical integer-order differential calculus. In fact, FC is an 'old' concept that was triggered by a question posed in a correspondence between Leibniz and liHôpital [1-3]. Nevertheless, FC is currently considered as a 'new' topic because during the last two decades relevant studies emerged in the areas of physics and engineering [4-11] motivating an increasing interest in its application. Also recently the concept of Power Law (PL) was observed in many physical, medical, economical and social phenomena [12-18]. Empirical approaches to PL dynamical evolutions were adopted, and ongoing research is presently starting to establish links between FC and PL.

The entropy was introduced in thermodynamics by Clausius and Boltzmann. Later the concept was applied by Shannon and Jaynes to information theory [19-21]. During the last years several alternative entropy measures were proposed, allowing the relaxation of the additivity axiom for application in several types of complex systems [22-29].

These concepts are presently under a large development and open up ambitious perspectives. Bearing these facts in mind, the present study takes advantage of the synergies associated with different, but complementary tools, towards the dynamical analysis of multi-particle systems with impact phenomena. The paper is organized as follows. Section 2 introduces the fundamentals of the fractional calculus and the entropy. Section 3 formulates the system conditions and develops a dynamical analysis in the viewpoint of fractional dynamics and entropy. Finally, Section 4 outlines the main conclusions.

\section{Preliminaries}

FC is a generalization of the ordinary integer differentiation and integration to an arbitrary order. The Riemann-Liouville, Grünwald-Letnikov, and Caputo definitions of fractional derivatives are given by:

$$
\begin{gathered}
{ }_{a} D_{t}^{\alpha} f(t)=\frac{1}{\Gamma(n-\alpha)} \frac{d^{n}}{d t^{n}} \int_{a}^{t} \frac{f(\tau)}{(t-\tau)^{\alpha-n+1}} d \tau \\
n-1<\alpha<n
\end{gathered}
$$




$$
\begin{aligned}
{ }_{a} D_{t}^{\alpha} f(t) & =\lim _{h \rightarrow 0} \frac{1}{h^{\alpha}} \sum_{k=0}^{\left[\frac{t-a]}{h}\right]}(-1)^{k}\left(\begin{array}{l}
\alpha \\
k
\end{array}\right) f(t-k h) \\
{ }_{a} D_{t}^{\alpha} f(t) & =\frac{1}{\Gamma(\alpha-n)} \int_{a}^{t} \frac{f^{(n)}(\tau)}{(t-\tau)^{\alpha-n+1}} d \tau \\
& n-1<\alpha<n
\end{aligned}
$$

where $\Gamma()$ is Euler's gamma function, $[x]$ means the integer part of $x$, and $h$ is the step time increment.

Using the Laplace transform we have the expression:

$$
\mathcal{L}\left\{{ }_{0} D_{t}^{\alpha} f(t)\right\}=s^{\alpha} \mathcal{L}\{f(t)\}-\sum_{k=0}^{n-1} s_{0}^{k} D_{t}^{\alpha-k-1} f\left(0^{+}\right)
$$

where $s$ and $\mathcal{L}$ represent the Laplace variable and operator, respectively. These expressions reveal that fractional derivatives capture the whole history of the variable, that is, they have a memory, contrary to integer derivatives which are merely local operators.

The Mittag-Leffler function $E_{\alpha}(x)$ is defined by:

$$
E_{\alpha}(x)=\sum_{k=0}^{\infty} \frac{x^{k}}{\Gamma(\alpha k+1)}
$$

and interpolates between a purely exponential law, for phenomena governed by ordinary integer order equations, and a PL like behavior, for phenomena with fractional dynamics. In particular, when $\alpha=1$ we have $E_{1}(x)=e^{x}$. An important characteristic of the Mittag-Leffler function is its asymptotic behavior and, for large values of $x$, we can write:

$$
E_{\alpha}(-x) \approx \frac{1}{\Gamma(1-\alpha)} \frac{1}{x}, \quad \alpha \neq 1
$$

The Laplace transform yields:

$$
\mathcal{L}\left\{E_{\alpha}\left( \pm a t^{\alpha}\right)\right\}=\frac{s^{\alpha-1}}{s^{\alpha} \mp a}
$$

Therefore, we verify a natural extension of the Laplace transform pairs for the exponential function, in terms of integer powers of $s$, to the Mittag-Leffler function, in terms of fractional powers of the transform parameter $s$.

PLs govern a wide variety of natural and human phenomena, including frequencies of words, earthquakes, wars, music, and many other quantities. Recent interest in PLs is associated with the study of probability distributions because it is now recognized that the distributions of a wide variety of quantities seem to follow the PL form, at least in their upper tail. In general, many alternative functional forms can appear to follow a PL for some extent and the validation of PLs models remains an active field of research in many areas of modern science.

The concept of entropy was developed by Ludwig Boltzmann during the 1870s when analyzing the statistical behavior of systems microscopic components. Entropy is often loosely associated with the amount of disorder in a thermodynamic system. In information theory, entropy was devised by Claude Shannon in 1948 to study the amount of information in a transmitted message.

The Shannon entropy $S$, that satisfies the so-called Shannon-Khinchin axioms, is defined as:

$$
S=-\sum_{i=1}^{N} p_{i} \ln \left(p_{i}\right)
$$

where $N$ represents the number of possible events and $p_{i}$ is the probability that event $i$ occurs, so that $\sum_{i=1}^{N} p_{i}=1$.

The Shannon entropy represents the expected value of the information $-\ln \left(p_{i}\right)$. For the uniform probability distribution we have $p_{i}=N^{-1}$ and the Shannon entropy takes its maximum value $S=\ln (N)$, yielding the Boltzmanns formula, up to a multiplicative factor $k$ denoting the Boltzmann constant. Therefore, in thermodynamic equilibrium, the Shannon entropy can be identified as thephysical entropy of the system.

Two generalizations of the entropy consist in the Rényie and Tsallis entropies given by:

$$
\begin{aligned}
S_{q}^{(R)} & =\frac{1}{1-q} \ln \left(\sum_{i=1}^{N} p_{i}^{q}\right) \\
S_{q}^{(T)} & \left.=\frac{1}{q-1} 1-\sum_{i=1}^{N} p_{i}^{q}\right)
\end{aligned}
$$

which reduce to the Shannon entropy when $q \rightarrow 1$. 
Recently [30] it was proposed the fractional entropy:

$$
S_{q}^{(U)}=\sum_{i=1}^{N}\left(-\ln p_{i}\right)^{q} p_{i}
$$

which has the same properties as the Shannon entropy except additivity. Other measures were proposed and, for example, we can mention as the Landsberg-Vedral, Abel, Kaniagakis and Sharma-Mital entropies.

\section{Dynamical analysis systems with impacts}

In this section is analyzed the dynamics of systems consisting of $n$ elements denoted as 'particles', that exhibit dynamical interactions between them, denoted as 'impacts'. On the other hand, the effects of containers or walls are not considered.

We adopt an isolated system where each particle has a one-dimensional space excursion $x_{i}(t), i=1, \ldots, n$ where $t$ represents the time. Particles have mass $M_{i}$ and follow Newton's classical law. Since it is considered that there is no driving force, in the absence of impacts we have for particle $i$ the individual dynamics:

$$
0=M_{i} \ddot{x}_{i}
$$

The existence of dynamical interaction corresponds to a collision between two distinct particles. The velocities particles $i$ and $j$ after the impact $\left(\dot{x}_{i}^{\prime}\right.$ and $\left.\dot{x}_{j}^{\prime}\right)$ are related with their values before the impact $\left(\dot{x}_{i}\right.$ and $\left.\dot{x}_{j}\right)$ through the expression:

$$
\dot{x}_{i}^{\prime}-\dot{x}_{j}^{\prime}=-\varepsilon\left(\dot{x}_{i}-\dot{x}_{j}\right), \quad 0 \leqslant \varepsilon \leqslant 1
$$

where $\varepsilon$ is the coefficient of restitution that represents the dynamic phenomenon occurring in the masses during the impact. In the case of a fully plastic (inelastic) collision $\varepsilon=0$, while in the elastic case $\varepsilon=1$.

The principle of conservation of momentum requires that the momentum, immediately before and after the impact, must be identical:

$$
M_{i} \dot{x}_{i}^{\prime}+M_{j} \dot{x}_{j}^{\prime}=M_{i} \dot{x}_{i}+M_{j} \dot{x}_{j}
$$

From Eqs. (13) and (14) we can find the velocities of both masses after an impact, yielding:

$$
\begin{aligned}
& \dot{x}_{i}^{\prime}=\frac{\dot{x}_{i}\left(M_{i}-\varepsilon M_{j}\right)+\dot{x}_{j}(1+\varepsilon) M_{j}}{M_{i}+M_{j}} \\
& \dot{x}_{j}^{\prime}=\frac{\dot{x}_{i}(1+\varepsilon) M_{i}+\dot{x}_{j}\left(M_{j}-\varepsilon M_{i}\right)}{M_{i}+M_{j}}
\end{aligned}
$$

The total kinetic energy loss $E_{L}$ at an impact is determined by:

$$
E_{L}=\frac{1-\varepsilon^{2}}{2} \frac{M_{i} M_{j}}{M_{i}+M_{j}}\left(\dot{x}_{i}-\dot{x}_{j}\right)^{2}
$$

In this paper are adopted $n=10^{4}$ identical particles (i.e., $M_{i}=M_{j}$ ) and the initial position and velocity of each particle are generated by a normalized Gaussian distribution with average $\mu=0$ and standard deviation $\sigma=10$.

The system state is measured through the total energy $E=\sum_{i=1}^{n} \dot{x}_{i}^{2}$ and, alternatively, through $S, S_{q}^{(R)}, S_{q}^{(T)}$ and $S_{q}^{(U)}$. For the Rényie, Tsallis and Ubriaco entropies are evaluated the cases $q=\left\{\frac{1}{2}, 2\right\}$. The probabilities are approximated through the relative frequencies of occurrence. For this purpose, in each time step $d t$, is constructed the histogram characterizing the particle velocities with 50 bins in the range $\left|\dot{x}_{i}\right| \leqslant \max \left(\left|\dot{x}_{i}\right|\right)$.

The impact between two distinct particles is considered to occur when their distance reaches a given threshold, that is, when $\left|x_{i}(t)-x_{j}(t)\right|<\Delta x_{\min }$, and the relative velocities is not close to zero, that is, when $\left|\dot{x}_{i}\left(t_{n} s c 10\right)-\dot{x}_{j}(t)\right|>\Delta \dot{x}_{\min }$. In the sequel are tested different particle diameters $\Delta x_{\min }$ for a fixed value $\Delta \dot{x}_{\min }=0.005$; moreover, $d t=10^{-3} \mathrm{~s}$ and $10^{3}$ time samples is adopted leading to a maximum simulation time of $t_{\max }=1.0 \mathrm{~s}$. Therefore, by applying a time-sliding window with duration $d t$, the energy and the entropy time evolution $E(t), S(t), S_{q}^{(R)}(t), S_{q}^{(T)}(t)$ and $S_{q}^{(U)}(t)$ are obtained [31], producing curves that depend on the system characteristics, the entropy formula and the time.

Fig. 1 shows the system's energy versus time for the cases $\varepsilon=\left\{\frac{1}{4}, \frac{1}{2}, \frac{3}{4}, 1\right\}, \Delta x_{\min }=10^{-2}$, while Fig. 2 presents the corresponding evolution of the different entropies for $\varepsilon=\frac{1}{2}$. Figs. 3 and 4 depict the system's energy and Shannon entropy versus time for the cases $\Delta x_{\min }=\left\{10^{-3}, 2 \cdot 10^{-3}, \ldots, 10 \cdot 10^{-3}\right\}$ when $\varepsilon=\frac{1}{2}$.

As expected we verify that, in all cases, the energy and the entropy decrease monotonically and reveal distinct phases, namely $(i)$ an initial fast transient, corresponding to the dissipation of the randomly generated initial conditions, (ii) the main part following a PL evolution (corresponding to the straight line in the log-log charts), that represents the dynamic interaction with impacts between the particles, and (iii) a final noisy behavior, that is due to numerical problems such as the finite number of particles and, particularly at the entropies, the bin approximation for the calculation of relative frequencies. Furthermore, we verify that the effect of $\varepsilon$ is relatively small, which is in accordance with previous results [32], while $\Delta x_{\min }$ has a more visible effect. Finally, the different entropy formulations have a similar behavior in the present dynamical system. 


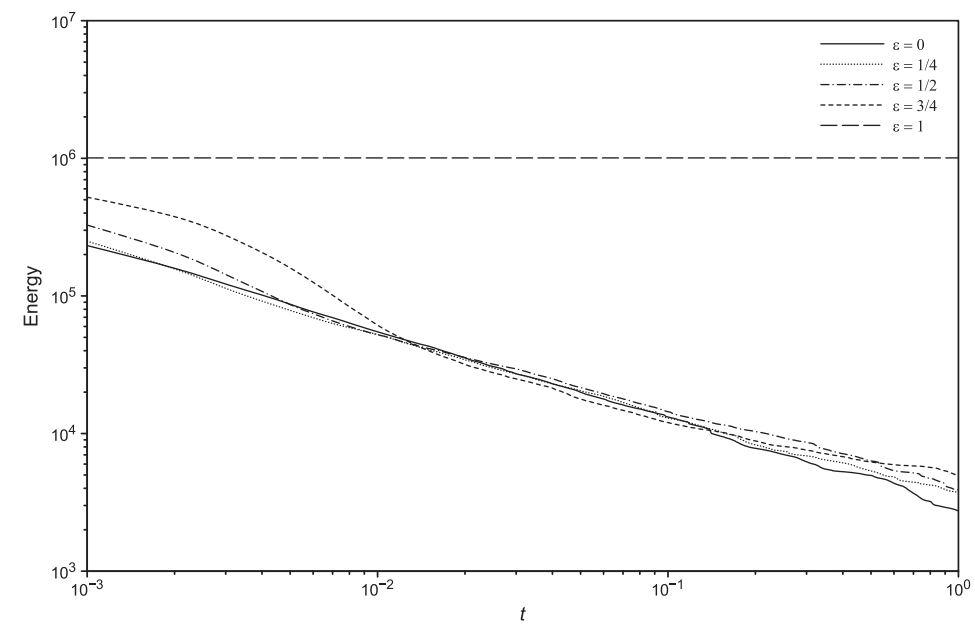

Fig. 1. Evolution of the energy $E(t)$ for systems composed by $n=10^{4}$ independent particles exhibiting impacts $\varepsilon=\left\{\frac{1}{4}, \frac{1}{2}, \frac{3}{4}, 1\right\}, \Delta x_{\min }=10^{-2}$.

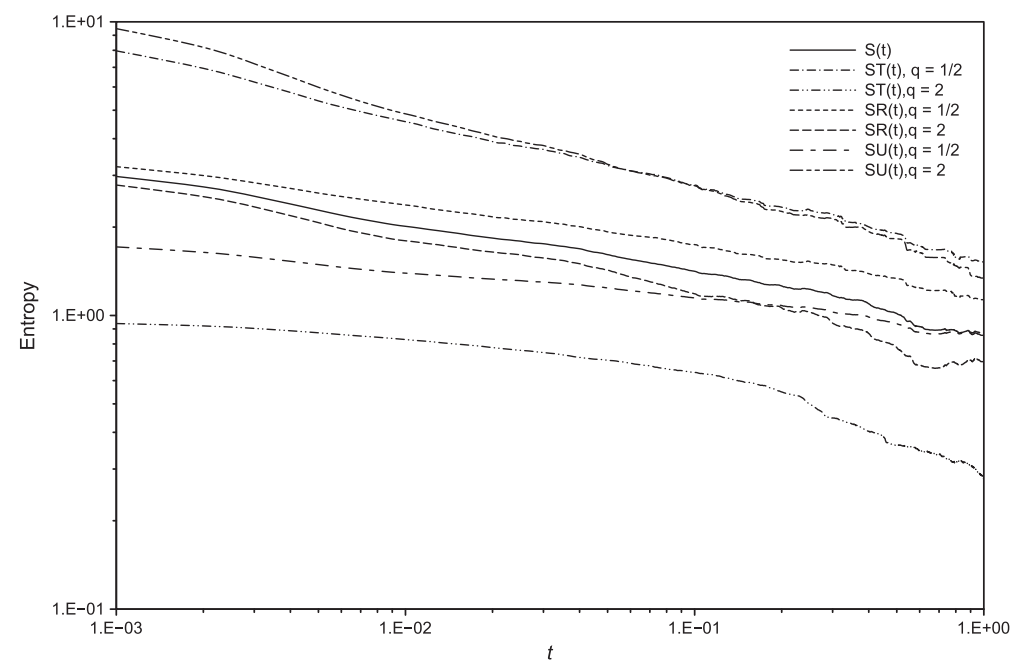

Fig. 2. Evolution of the entropies $S(t), S_{q}^{(R)}(t), S_{q}^{(T)}(t)$ and $S_{q}^{(U)}(t)$ for $q=\left\{\frac{1}{2}, 2\right\}$ for systems composed by $n=10^{4}$ independent particles exhibiting impacts $\varepsilon=\frac{1}{2}$, $\Delta x_{\min }=10^{-2}$.

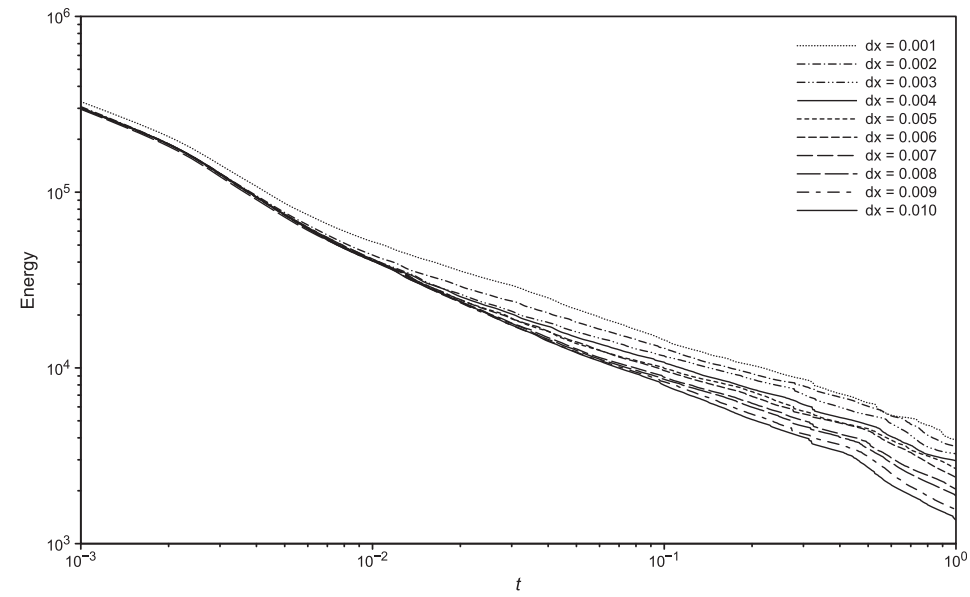

Fig. 3. Evolution of the energy $E(t)$ for systems composed by $n=10^{4}$ independent particles exhibiting impacts $\varepsilon=\frac{1}{2}, \Delta x_{\min }=\left\{10^{-3}, 2 \cdot 10^{-3}, \ldots, 10 \cdot 10^{-3}\right\}$. 


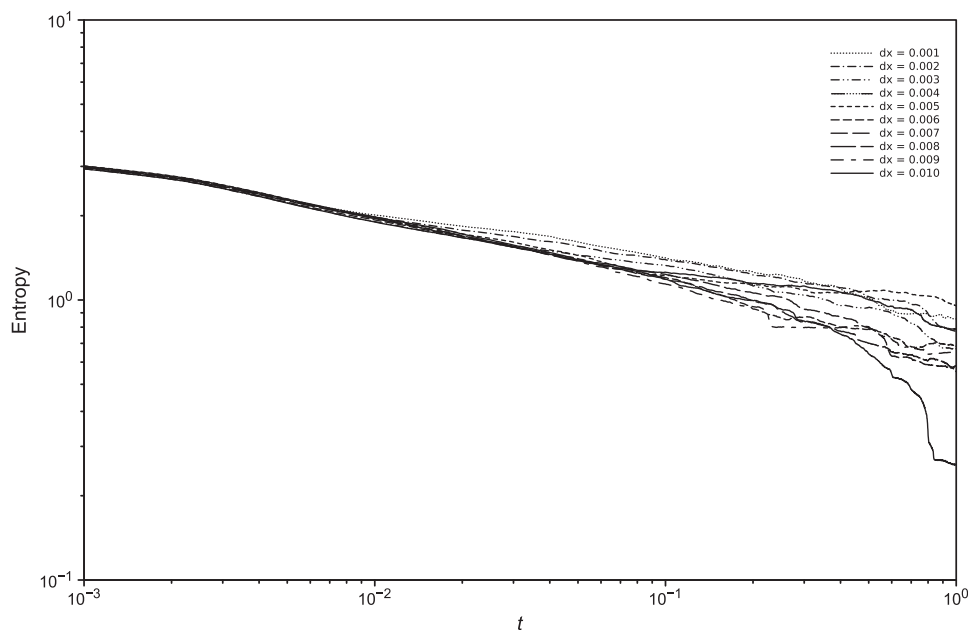

Fig. 4. Evolution of the Shannon entropy $S(t)$ for systems composed by $n=10^{4}$ independent particles exhibiting impacts $\varepsilon=\frac{1}{2}$, $\Delta x_{\min }=\left\{10^{-3}, 2 \cdot 10^{-3}, \ldots, 10 \cdot 10^{-3}\right\}$.

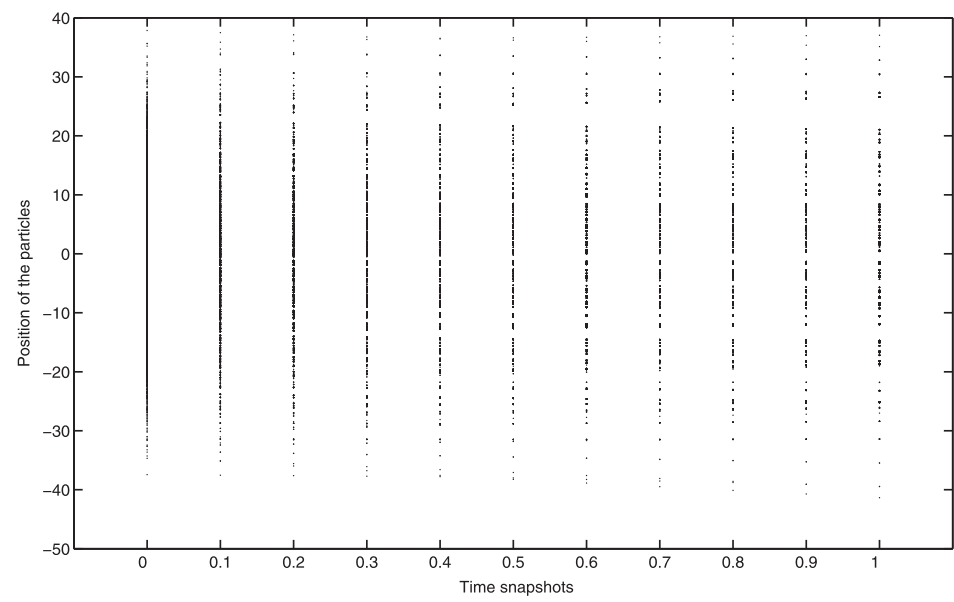

Fig. 5. Time snapshots of the particle positions for $\varepsilon=\frac{1}{2}, \Delta x_{\min }=10^{-2}$.

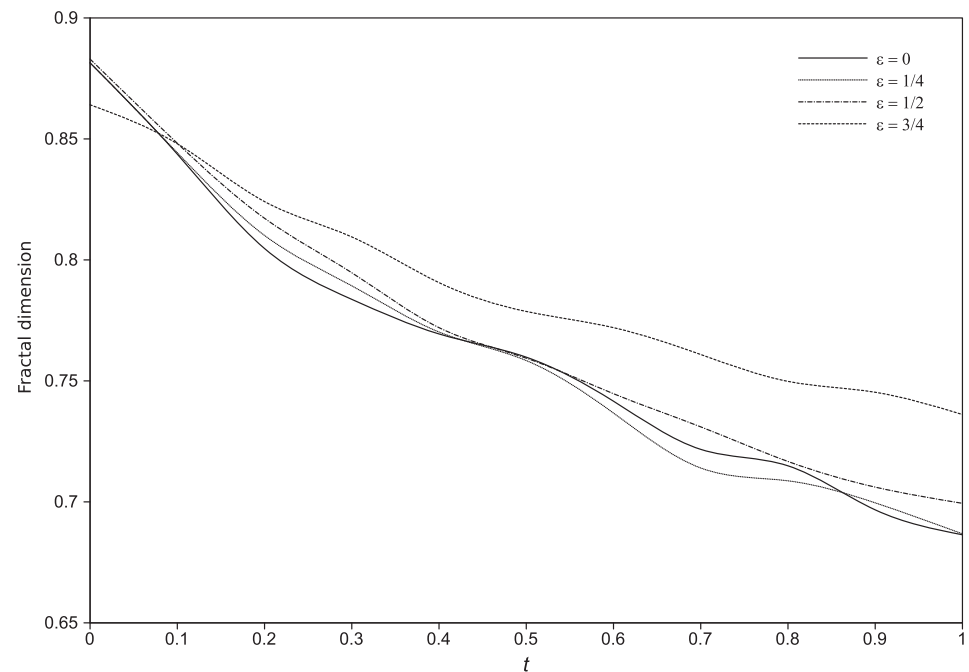

Fig. 6. Evolution of fractal dimension for $\varepsilon=\left\{0, \frac{1}{4}, \frac{1}{2}, \frac{3}{4}\right\}, \Delta x_{\min }=10^{-2}$. 
The time evolution of the energy and entropies plotted in the charts can be approximated with good accuracy by several functions. Nevertheless, to reveal more clearly the PL behavior, that is, to highlight the dynamics without considering the initial (transient) and final (noise signal) periods, the main dynamics was approximated numerically by the expressions $E(t) \sim t^{-\alpha}, \alpha>0$ and $S(t) \sim t^{-\beta}, \beta>0$. The PL approximations for the plots of Figs. 3 and 4 lead to $0.45 \leqslant \alpha \leqslant 0.63$ and $0.13 \leqslant \beta \leqslant 0.31$, respectively, and larger values the larger is the parameter $\Delta x_{\text {min }}$.

Another aspect of interest in the emergence of groups of particles as the results of the dynamic interaction between them. Fig. 5 depicts ten time snapshots of the particle positions, when $\varepsilon=\frac{1}{2}$. We observe that the almost continuous line at the beginning is gradually substituted by clusters with a clear fractal geometry. In this perspective, Fig. 6 shows the evolution of the fractal dimension versus time when $\varepsilon=\left\{0, \frac{1}{4}, \frac{1}{2}, \frac{3}{4}\right\}, \Delta x_{\min }=10^{-2}$ revealing that, as expected, the fractal dimension diminishes continuously.

In conclusion, the results reveal the emergence of fractional dynamics due to the interactions of a complex system modeled by classical integer-order dynamics, which is accordance with recent studies [32,33] connecting integral and fractional order models.

\section{Conclusions}

This paper reviewed two important mathematical tools, namely the fractional calculus and the entropy. These concepts allow a fruitful interplay in the analysis of system dynamics. Nevertheless, the synergies of applying both tools has been, somehow, neglected in engineering and applied sciences. The paper analyzed multi-particle systems with integer and fractional order behavior and demonstrated that the concepts are simple and straightforward to apply. In this line of thought, future research will address the analysis of more complex systems.

\section{References}

[1] Keith JS, Oldham B. The fractional calculus: theory and application of differentiation and integration to arbitrary order. New York: Academic Press; 1974.

[2] Podlubny I. Fractional differential equations, volume 198: an introduction to fractional derivatives, fractional differential equations, to methods of their solution. Math Sci Eng. San Diego: Academic Press; 1998.

[3] Kilbas HMSAA, Trujillo JJ. Theory and applications of fractional differential equations, volume 204. North-Holland mathematics studies. Amsterdam: Elsevier; 2006.

[4] Mainardi F. Chaos, Solitons Fractals 1996;7:1461-77.

[5] Machado JAT. Syst Anal Model Simul 1997;27:107-22.

[6] Nigmatullin RR. Signal Process 2006;86:2529-47.

[7] Baleanu D. Commun Nonlinear Sci Numer Simul 2009;14:2520-3.

[8] Podlubny I. IEEE Trans Automat Control 1999;44:208-13.

[9] Machado JAT. Fract Calculus Appl Anal 2001;4:47-66.

[10] Chen KLMYQ. IEEE Trans Circuits Syst-I: Fundam Theor Appl 2002;49:363-7.

[11] Tseng CC. IEEE Signal Process Lett 2001;8:77-9.

[12] Gutenberg CFRB. Seismicity of the Earth and Associated Phenomena. Princeton, N.J.: Princeton University Press; 1954.

[13] Bak KWP, Tang C. Phys Rev Lett 1987;59:381-4.

[14] Gabaix X. Quarter J Econ 1999;114:739-67.

[15] Li W. IEEE Trans Inform Theor 1992;38:1842-5.

[16] Egghe L. J Amer Soc Inform Sci Technol 2005;56:664-8.

[17] Newman MEJ. Contemp Phys 2006;46:323-51.

[18] Mollick E. IEEE Ann History Comput 2006;28:62-75.

[19] Shannon CE. Bell Syst Tech J 1948;27:379-423. pp. 623-656.

[20] Jaynes ET. Phys Rev 1957;106:620-30.

[21] Khinchin AI. Mathematical foundations of information theory. New York: Dover; 1957.

[22] Plastino ARPA. Brazilian J Phys 1999;29:50-60.

[23] Li MDKHX, Essex C, Schulzky C. J Non-Equilibrium Thermodyn 2003;28:279-91.

[24] Haubold AMMHJ, Saxena RK. Astrophys Space Sci 2004;290:241-5.

[25] Mathai HJHAM. Physica A: Stat Mech Appl 2007;375:110-22.

[26] T. Carter, An introduction to information theory and entropy, Complex Systems Summer School, Santa Fe, 2007.

[27] Rathie SdSP. Appl Math Sci 2008;2:1359-63.

[28] Beck C. Contemp Phys 2009;50:495-510.

[29] Gray RM. Entropy and information theory. New York: Springer-Verlag; 2009.

[30] Ubriaco MR. Phys Lett A 2009;373:2516-9.

[31] Machado JAT. Nonlinear Dyn 2010.

[32] Tenreiro Machado AMSGJA. ASME J Comput Nonlinear Dyn 2008;3. pp. 021201-1-021201-5.

[33] Nigmatullin DBRR. Int J Theor Phys 2010;49:701-8. 